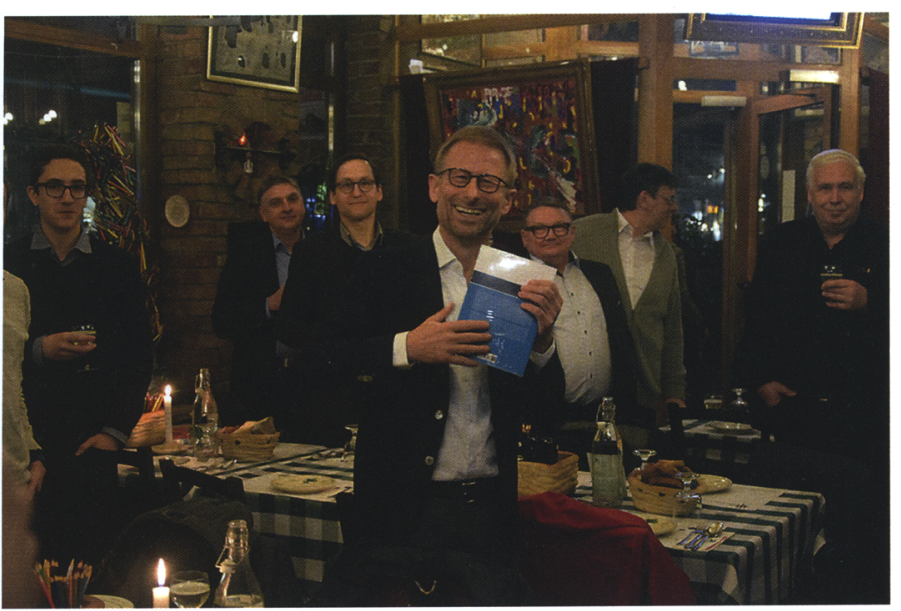

Prof. Asche erhält ein Buchgeschenk zur Emeritierung. Foto: Harald Schernthanner

Weltraumbild-Atlas“. Es folgten Stationen in der Fachgruppe Geographie/Geowissenschaften der Universität Trier und einer Universitätsassistenz am Ordinariat für Geographie und Kartographie des Instituts für Geographie der Universität Wien. 1986 bekam Herr Asche einen Ruf als Professor für Kartographie an den Fachbereich Vermessungswesen und Kartographie der Hochschule für Technik Karlsruhe. Diesen Lehrstuhl hatte er bis 1988 inne. Es folgte ein Ruf nach Berlin an die Beuth Hochschule, wo er die Professur für Kartographie am Fachbereich Vermessungs- und Kartenwesen annahm. In dieser Zeit (1988 bis 1995) leitete er das Labor für Geovisualisierung und Geoinformation der Beuth Hochschule. 1995 kam sein Ruf, als Universitätsprofessor für Geoinformatik, Fernerkundung und Kartographie an das Institut für Geographie der Universität Potsdam zu wechseln, an den Lehrstuhl, den Herr Asche bis zu seiner Emeritierung innehatte. In diesen Karriereabschnitt fallen zahlreiche große Erfolge seiner wissenschaftlichen Karriere, darunter über 40 referierte wissenschaftliche Artikel in den Feldern Kartographie, Geoinformation und Fernerkundung. Einer der Meilensteine aus Herrn Asches Potsdamer Zeit ist der Aufbau des Masterstudiengangs Geoinformation und Visualisierung. Der Studiengang basiert auf einem Curriculum der klassischen GI-Science, eingebettet in die Visualierungspipeline, ein Ansatz, der bis dato so nicht in der deutschsprachigen Geoinformationswelt zu finden war.

Ein weiterer Meilenstein ist der von 2001 bis 2003 entstandene bevölkerungsgeographische Atlas von Albanien. Dessen neuartige kartographische Produktion mittels Vektorgrafikprogrammen und GIS-Software war weit vor seiner Zeit. Die rezente Forschungstätigkeit von Herrn Asche ist im Bereich der Fußgängernavigation angesiedelt. Diese Forschungstätigkeit wird Herr Asche auch nach seiner Emeritierung fortführen.

In der Zeit von 1995 bis 2016 führte Herr Asche zahlreiche Doktoranden als Doktorvater zur Promotion. Viele seiner Schüler führen den von Herrn Asche geprägten Stil der Wissenschaft in ihrer Tätigkeit, unter anderem bei ESRI, Oracle, Immobilienscout24, Univerisity of Regina, weiter.

Nach der Laudatio wurde Herrn Asche eine von Mitarbeitern des Institutes für Geographie der Universität Potsdam herausgegebene Festschrift, als würdige Gabe anlässlich des formalen Endes seiner akademischen Karriere, überreicht. Die im Universitätsverlag im Rahmen der Reihe Potsdamer Geographische Praxis veröffentlichte Festschrift trägt den Titel „Geoinformation \& Visualisierung: Pionier und Wegbereiter eines neuen Verständnisses von Kartographie und Geoinfor- matik“. Die Festschrift umreißt die Schwerpunkte, mit welchen Herr Asche sich in seiner von zahlreichen Höhepunkten geprägten wissenschaftlichen Karriere beschäftigte. Sie ist unter der URL http://verlag.ub.unipotsdam.de/cgi-bin/publika/ view.pl?id=926 bezieh- und downloadbar.

Herr Asche antwortete auf die Laudatio in einem sehr persönlichen Redebeitrag, in dem er herausstrich, dass in seiner Karriere vor allem die Menschen, nicht so sehr die Karrierehöhepunkte, eine Bedeutung hatten, und er würdigte all jene, die ihn während seiner akademischen Karriere begleitet und geprägt haben. Herr Volkmar Scholz, der Lauftrainer des begeisterten Hobbyläufers Herrn Asche vom Berliner Laufverein „Die Laufpartner“, rundete die Redebeiträge mit einer sehr persönlichen Note ab. Danach wurde bei guter Pizza und gutem Wein bis weit nach Mitternacht geredet und gefeiert.

Harald Schernthanner,

Potsdam

\section{- Parlamentarisches Mittagessen im Bundestag}

Abgeordnete des Bundestages treffen sich mit Vertretern der Geoverbände

Lobbyismus im Bundestag hat in der öffentlichen Wahrnehmung einen fahlen Beigeschmack. Sehr schnell entsteht der Eindruck der Verfolgung einzelner Interessen oder Vorteilsnahme. Mit gutem Gewissen können dies jene Beteiligte aus den Geoverbänden von sich weisen, die sich am 8. März 2017 in Berlin mit Bundestagsabgeordneten getroffen haben. Keine der teilnehmenden Firmen oder Interessenverbände konnte auf eine vorteilhafte Würdigung der politischen Entscheidungsträger hoffen. Der Austausch stand allein unter dem Ziel, die gesamte Branche zu fördern, indem in der Politik die besondere Bedeutung von Geoinformationen in Wirtschaft und Gesellschaft gestärkt werden soll. Die Geoinformationswirtschaft - ein Motor der Digitalisierung war das Credo des einladenden Verbandes GEOkomm e. V., Verband der GeoInformationswirtschaft Berlin/ Brandenburg, dem für die Initiative und die hervorragende Organisation $\mathrm{zu}$ danken ist. GEOkomm ist insbesondere dafür zu danken, dass hier die richtigen Stakeholder mit den richtigen Politikern in Verbindung kommen. Als wichtigste Verbände waren neben der DGfK auch DVW, BDVI, VDV, DDGI und DMV vertreten. Aus der Bundespolitik waren anwesend: Staatssekretärin $\mathrm{MdB}$ Dorothee Bär (CSU), Bundesministerium für Verkehr und digitale Infrastruktur, der wissenschaftliche Mitarbeiter Simon Weiß, Büro Dr. Petra Sitte, Erste Parlamentarische Geschäftsführerin Fraktion DIE LINKE, MdB DR. Peter Ramsauer, Deutscher Bundestag, Aus-

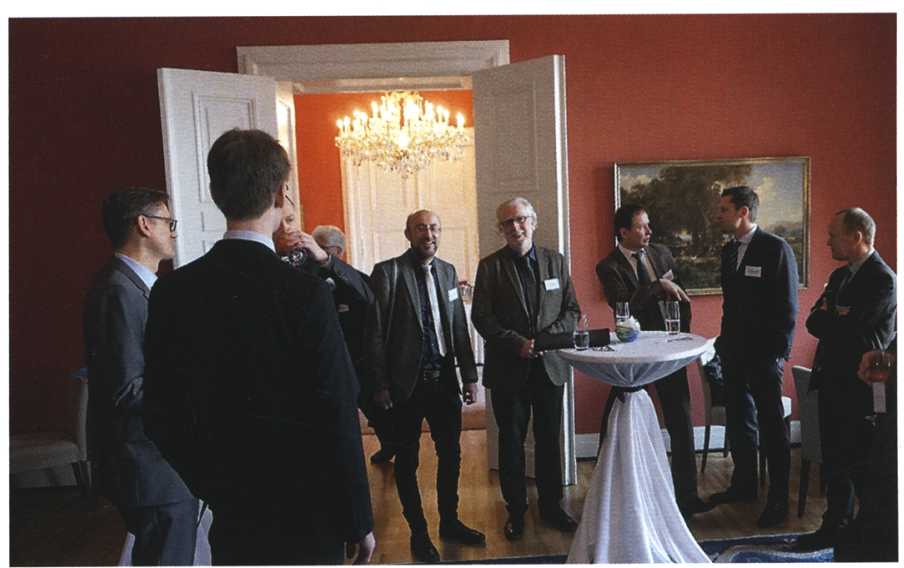

Abb. 1: Teilnehmende am Parlamentarischen Mittagessen. Foto: Mark Vetter 


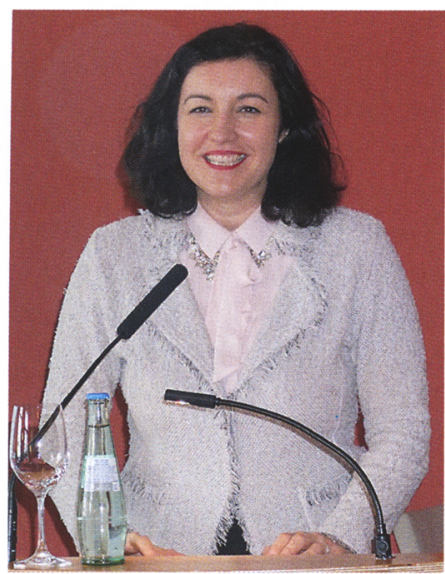

Abb. 2: Die Staatssekretärin vom BMVI Dorothee Bär (CSU), hält das Grußwort zum Parlamentarischen Mittagessen in Berlin. Foto: Mark Vetter

schuss Wirtschaft \& Energie, MdB Florian Oßner (CSU), Ausschuss für Verkehr und digitale Infrastruktur.

Zur Begrüßung der Veranstaltung hält Dr. Peter A. Hecker, Vorstandsvorsitzender GEOkomm, ein Plädoyer zur gegenwärtigen Bedeutung von Geoinformationen, nicht nur aus der ökonomischen Perspektive, sondern er stellt heraus, dass auch in der Zivilgesellschaft Geoinformationen in der Medizin oder im Katastrophenschutz lebensrettend sein können. Schon in der Einladung zur Veranstaltung führt Herr Hecker aus, dass ortsbezogene Informationen in Form von Geodaten als digitaler Rohstoff mittlerweile in fast allen wirtschaftlichen und gesellschaftlichen Bereiche Eingang gefun-

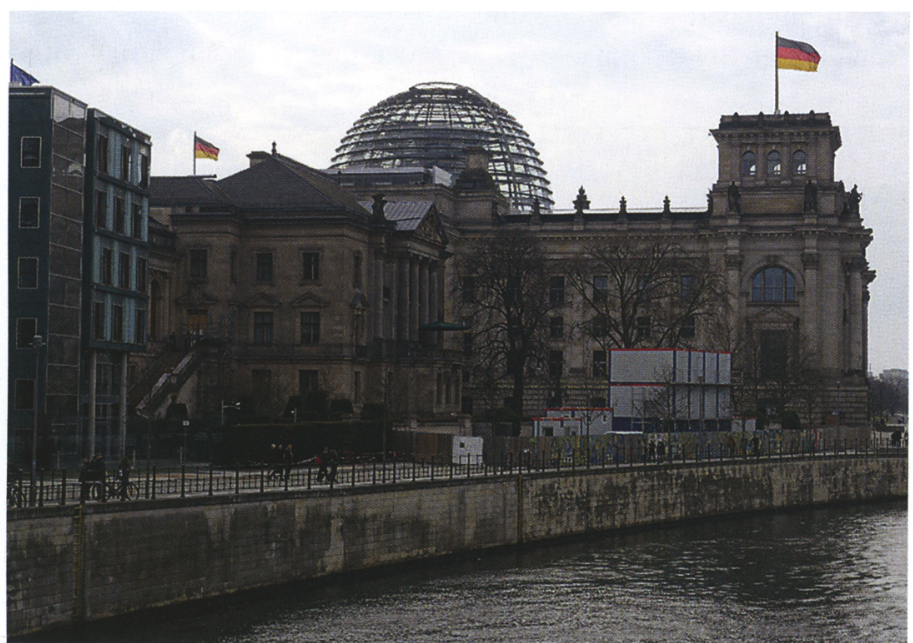

Abb. 3: In Bildmitte links ist ein Altbau zu sehen. Dies ist der Veranstaltungsort, das Haus der Parlamentarischen Gesellschaft. Foto: Mark Vetter den haben. Geoinformationen spielen eine wichtige Rolle in Unternehmensprozessen. Dabei sind sie ein wesentlicher Baustein der Digitalisierung, der aufgrund seiner Querschnittsfunktion zu einer ganzheitlichen Informations- und Kommunikationsinfrastruktur beitragen wird. Er betont, dass durch neue gesellschaftliche Anforderungen in Verbindung mit dem technologischen Fortschritt, z. B. in den Bereichen Energiewende und Mobilität, Geoinformationen einen weiteren erheblichen Bedeutungsgewinn erfahren werden. Verfügbarkeit und Zugänglichkeit des Rohstoffs Geoinformation sind Voraussetzungen für die Initiierung von Wertschöpfungsprozessen sowie die Umsetzung und Optimierung von Digitalisierungsprozessen.

Das Grußwort zur Bedeutung der Geoinformationswirtschaft im Zuge der Digitalisierung hält die Staatssekretärin im BMVI Dorothee Bär. Sie ergänzt, dass die Geoinformationen nicht nur für den Motor der Digitalisierung wichtig sind, sondern auch gleichermaßen Treibstoff dieser Entwicklung sind. Sie berichtet voller Stolz über die Aktivitäten im BMVI und bedauert, dass das Ministerium über die MautDiskussion ungerechtfertigterweise so viel negative Publicity bekommt. Frau Bär hob die Innovationskraft, die vom satellitenbasierten, hochpräzisen empfunden. Es wurde darauf hingewiesen, dass der Mehrwert von Geoinformationen noch stärker in der Politik verankert werden muss. Diese Veranstaltung sol wiederholt werden, vermutlich aber erst nach der Bundestagswahl. Als teilnehmende Bundespolitikerinnen oder Bundespolitiker sollen dann Vertreter aus dem Wirtschaftsministerium eingeladen werden. Die Wirtschaftszweige Energie und Geoinformationen sollen dann im Fokus des parlamentarischen Mittagessens stehen.

Mark Vetter, Karlsruhe
IfL kartiert Nesthocker

Vor allem in Deutschlands Süden verlassen junge Erwachsene erst spät die elterlichen vier Wände, wie aktuelle Karten des LeibnizInstituts für Länderkunde zeigen.

In Thüringen, Bayern und im Saarland lebt mehr als ein Viertel der 25- bis 29-Jährigen noch zuhause bei den Eltern. Spitzenwerte erreicht die Nesthocker-Quote in den bayerischen Landkreisen Freyung-Grafenau und Straubing-Bogen sowie im thüringischen Eichsfeld. Dort leben in dieser Altersgruppe die Hälfte der Männer und fast ein Drittel der Frauen noch im Elternhaus. In den Stadtstaaten und in Schleswig-Holstein logieren dagegen nur zwischen zehn und 15 Prozent der jungen Erwachsenen im Hotel Mama. Auffällig ist in allen Bundesländern der deutliche Stadt-Land-Unterschied. Das liegt vor allem an der Konzentration der Hochschulen in den Städten, denn die Aufnahme eines Studiums ist ein wichtiger Auszugsgrund. Auszubildende bleiben dagegen häufig so lange im Elternhaus, bis sie einen festen Job haben oder eine eigene Familie gründen. Mit deutschlandweit 26 Prozent ist der Anteil der jungen Männer, die noch im Haushalt der Eltern leben, fast doppelt so hoch wie derjenige der jungen Frauen.

$\mathrm{Zu}$ diesen Ergebnissen kommt Dr. Tim Leibert vom Leibniz-Institut für Länderkunde (IfL). Der Bevölkerungsgeograph hat für das Webangebot "Nationalatlas aktuell“ des IfL die Statistiken zu Haushalten und Familien erstmals auf Basis der Kreise und kreisfreien Städte ausgewertet und interpretiert. Aktuelle Karten des IfL veranschaulichen die regionalen Unterschiede.

Der vollständige Beitrag ist im Webangebot „Nationalatlas aktuell“ des IfL nachzulesen. Auf http://aktuell.nationalatlas.de veröffentlicht das Institut regelmäßig Kartenbeiträge zu Themen aus Wirtschaft, Gesellschaft, Kul- 